\title{
Land use and pollution in a two-sector evolutionary model
}

\author{
Angelo Antoci ${ }^{\mathrm{a}}$, Simone Borghesi ${ }^{\mathrm{b}, \mathrm{c}}$, Gianluca Iannucci $^{\mathrm{d}, *}$, Elisa Ticci $^{\mathrm{c}}$

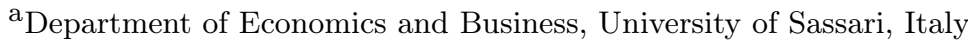 \\ ${ }^{\mathrm{b}}$ FSR Climate, European University Institute, Florence, Italy \\ ${ }^{\mathrm{c}}$ Department of Political and International Studies, University of Siena, Italy \\ ${ }^{\mathrm{d}}$ Department of Economics and Management, University of Florence, Italy
}

Accepted for publications in Structural Change and Economic Dynamics

\begin{abstract}
This paper proposes an evolutionary dual model in order to study heterogeneity of agriculture from an environmental perspective. In standard visions, land use changes are driven by productivity differentials across agricultural subsectors. Our model adds other two factors which can indirectly affect land allocation patterns: differences in environmental impact intensity and environmental vulnerability. By including these two dimensions in a parsimonious model, we shed new light on constraints or ability for an economy to reach a sectoral land allocation which efficiently balances environmental burden, environmental constraints and productivity.

We find that, in case of trade-off between the three dimensions, farmers' welfare grows along structural changes which allocate increasingly more land to the agricultural subsector with lower pollution intensity regardless its resilience and land productivity performance.

Keywords: Agricultural subsectors, Evolutionary model, Environmental externalities, Vulnerability to pollution.

${ }^{*}$ Corresponding author. Via delle Pandette 9, 50127 Firenze, Italy.

E-mail addresses: antoci@uniss.it (A. Antoci), simone.borghesi@eui.eu / simone.borghesi@unisi.it (S. Borghesi), gianluca.iannucci@unifi.it (G. Iannucci), ticci4@unisi.it (E. Ticci).
\end{abstract}




\section{Introduction}

The important role of agriculture for overall socio-economic development and for poverty reduction has been largely acknowledged by development theory for decades (Johnston and Mellor, 1961; Adelman, 1984; Vogel, 1994) and it has been recently confirmed by further studies (De Janvry and Sadoulet, 2010; Christiaensen et al., 2011; Ligon and Sadoulet, 2018). The mostly used conceptual framework compares agriculture with non agricultural activities even if agriculture is not a monolithic sector. Indeed, dualism in the agriculture sector is not a new topic but, all over the world, agriculture has been experiencing important evolutions which go beyond the standard dichotomy between traditional and mechanized modern farming. Heterogeneity of agriculture both in high and low income countries involves several dimensions, from type of organizational models, degree of integration in international markets to technological content. Empirical studies show that heterogeneity across agricultural subsectors matters since not all agricultural activities have the same potential for the economic development of countries. Diao et al. (2012), for instance, show that cultivation of food staples is usually more effective than export agriculture at promoting economic growth and poverty reduction. Other authors have investigated the welfare effect of different organizational models, such as contract farming compared to independent smallholder farming (Bellemare, 2012) or outgrower schemes compared to plantations (Arndt et al., 2010).

Farming practices can differ also in terms of resilience to shocks, demand of natural resources and creation of environmental damage, but our understanding on agriculture's heterogeneity from an environmental viewpoint is less advanced, though not less relevant. In a context of growing pollution, resource depletion and climate change manifestations, the success of development is conditional on its environmental impact (De Janvry and Sadoulet, 2017). Thus, the environmental perspective cannot be overlooked and agriculture is part of this debate since this sector, not only has a big power for poverty reduction and economic development, but is also a major user (or misuser) of natural resources.

However, the distinction of agriculture subsectors by environmental burden or constraints is an intrinsically difficult exercise further compounded by lack of data. In absence of representative dataset and evidence, theoretical models, in combination with case studies, can be 
effective at delineating possible dynamic regimes emerging in the inextricable nexus between agriculture composition, environment and economic development. This is the aim of this work. We contribute to the debate on differential and dynamic effects on agricultural subsectors by concentrating on environmental linkages. This focus is the first novelty of this work but a caveat is required: the model does not substitute, it complements analyses with backward and forward economic linkages which here are excluded for reasons of analytical tractability. Secondly, we focus on coordination problems in a context of environmental externalities. Despite the consensus on the importance of environmental regulation and valuation of nature and ecosystem services, markets for environmental quality in agriculture are usually incomplete or lacking. Many natural resources and ecosystem services have the character of public goods (air quality, rivers, free access forests, fish stocks, genetic diversity, environmental regulating services) and, for many environmental resources, the creation and enforcement of clear property or use rights is very difficult. As a consequence, choice processes in farming are often taken in a context of shallow, nonexistent or incomplete markets with pervasive externalities. Farmers' decisions usually do not fully consider the environmental impact of agricultural practices. For instance they rarely pay for discharge of pollutants and sediment into the environment or for soil erosion, degradation or salinization due to poor management practices. These environmental damages do not come without economic consequences on agricultural activities themselves. Pesticides, herbicides, fertilizers and other agrochemicals, for instance, can generate a long list of detrimental effects on productivity of either "polluting" farms or other "victim" farms. Examples include destruction of natural enemies of pests (Lichtenberg, 2002; Pelletier and Tyedmers, 2010), development of pesticide resistance, crop pollination problems, honeybee losses, biodiversity ${ }^{1}$ loss (Baudron and Giller, 2014) that deteriorate the stability and resilience of agricultural systems (see, e.g., Brittain et al., 2013), or even labour productivity loss due to the impact on farm workers' health. This feedback effects can bring about mechanisms of perverse self-reinforcing sector specialization and crowding out effects. To fix ideas, we can mention two examples, a global and a local one. Let us consider the coexistence of sustainable forestry activities and agriculture. The impact of climate change on agriculture productivity is predominantly negative, particularly in low-income coun-

\footnotetext{
${ }^{1}$ The importance of biodiversity management is discussed in several contributions in economic literature (see, e.g., Rauscher and Barbier, 2010; Vardas and Xepapadeas, 2010).
} 
tries that have higher climate vulnerability and lower adaptation capital stocks, which hinders their prospects for development (Bretschger and Valente, 2011; Bretschger and Suphaphiphat, 2014). However, the main options that this sector uses to counteract to it - intensification or land-use change - further increases greenhouse gas emissions. This process with self-reinforcing nature (Bajželj and Richards, 2014) can be associated with crowding out of the sector less responsible for climate change since agricultural land expansion is likely to happen at the expense of forestry activities. Another example of a welfare-reducing specialization and crowding-out mechanism is related to the ecology of pollinators. Crop rotation, organic farming (Gabriel and Tscharntke, 2007; Bengtsson et al., 2005), patches with high plant diversity within farmland (Gigante Carvalheiro et al., 2011) have beneficial effects on pollinators. However, farmers rarely adopt these practices, instead preferring conventional farming and large-scale agricultural intensification, removing naturally occurring plants. The expansion of these agricultural practices reduces the productivity in all sectors depending on pollination services and may give rise to an inefficient specialization and productivity losses. Other examples will be discussed later.

Based on this background, we propose a two-sector evolutionary model in which economic agents have to allocate land between two agricultural sectors, $A$ and $B$. For both sectors, land is the only choice variable and production is damaged by environmental effects generated by the agricultural activities themselves. At the same time, sectors $A$ and $B$ differ by production elasticity with respect to land, degree of environmental burden and by vulnerability to environmental degradation. Following IPCC (IPCC Glossary, 2014, p.218), vulnerability can be defined as the "propensity or predisposition to be adversely affected", a multidimensional notion which "encompasses a variety of concepts including sensitivity or susceptibility to harm and lack of capacity to cope and adapt". To better focus the concept and apply it in the present analysis, by 'vulnerability to environmental degradation' we will mean here the adverse impact that higher environmental degradation (measured by pollution in the model) has on the production level of each sector. ${ }^{2}$

The effects of environmental impact are not internalized by the market (i.e. by prices). The model is thus very essential but it generates multiple environment-sector compositiondevelopment patterns providing non trivial, even unexpected, results. In particular we show

\footnotetext{
${ }^{2}$ For a deeper discussion on the existing definitions of vulnerability see weadapt.org.
} 
under what conditions the reallocation choices of land endowment from one sector to the other one may give rise to a welfare-reducing specialization characterized by a reduction in farmers' average revenues and by uniformity of landscape.

The rest of the paper is organized as follows. Section 2 presents the model, Section 3 carries out the analysis of stability properties of stationary states, while Section 4 proposes a classification of possible dynamic regimes produced by the model. Section 5 performs some exercises of comparative statics on parameters representing two dimensions of interest, namely vulnerability to pollution and land productivity. Section 6 discusses the socio-economic relevance of the analytical results and Section 7 concludes.

\section{The model}

We model a small open economy with two agricultural sectors, $A$ and $B$, in which all prices are exogenously set. Land endowment, measured by the parameter $\bar{L}>0$, is fixed; $L_{A}$ and $L_{B}$, $L_{A}+L_{B}=\bar{L}$, indicate the amount of land (measured, e.g., in hectares) devoted to production activities of sector $A$ and $B$. Aggregate outputs of sectors $A$ and $B$ are given respectively by the constant returns functions: ${ }^{3}$

$$
\begin{aligned}
& Y_{A}=\alpha L_{A} \\
& Y_{B}=\beta L_{B}
\end{aligned}
$$

where the parameters $\alpha>0$ and $\beta>0$ measure the production elasticities with respect to land (i.e. they are indicators of land productivity) in sectors $A$ and $B$, respectively. To simplify, we assume that the prices of the goods produced in sectors $A$ and $B$ are both equal to unity. So $Y_{A}$ and $Y_{B}$ also represent the market value of aggregate outputs of sectors $A$ and $B$, respectively. ${ }^{4}$

Indicating with the variable $x \in[0,1]$ the share of land endowment $\bar{L}$ used by sector $A$ (i.e.,

\footnotetext{
${ }^{3}$ We can consider the following production functions as deriving from a Leontief function of the type $Y=$ $\min (a L, b K, c W)$, where $K$ and $W$ represent physical capital and labor, while $a, b, c>0$ are parameters. Supposing that physical capital and labor are not constrained (namely, land is the only scarce factor), then $K$ and $W$ are chosen to satisfy $a L=b K=c W$, from which $K=a L / b$ and $W=a L / c$. Assuming that both inputs prices, $r$ (physical capital) and $w$ (labor), and output price $p$ of sector $A$ are constant and exogenously given (small open economy hypothesis), then the aggregate profits can be rewritten as: $\pi=p a L-r(a / b)-w(a / c)=a(p-r / b-w / c) L$. Setting $\alpha=a(p-r / b-w / c)$, considering the negative effect of pollution and applying the same to sector $B$, we obtain functions (1).

${ }^{4}$ The results of the model hold true even if we relax the assumption of equal goods' prices in the two sectors. In fact, $\alpha$ and $\beta$ can be considered as composite parameters that measure possible differences between sectors $\mathrm{A}$ and $\mathrm{B}$ in both land productivity and prices.
} 
$L_{A}=x \bar{L}$ ) and with $1-x$ the share of $\bar{L}$ used by sector $B$ (i.e., $L_{B}=(1-x) \bar{L}$ ), the production functions (1) can be rewritten as follows:

$$
\begin{gathered}
Y_{A}=\alpha x \bar{L} \\
Y_{B}=\beta(1-x) \bar{L}
\end{gathered}
$$

The production activities of both sectors determine the time evolution of the value of a pollution index, $P \in[0,+\infty)$, according to the equation:

$$
\dot{P}=\varepsilon \alpha x \bar{L}+\eta \beta(1-x) \bar{L}-\theta P
$$

where $\dot{P}$ is the time derivative of $P$, the parameters $\varepsilon \geq 0$ and $\eta \geq 0$ measure the impact on the pollution accumulation process of the aggregate outputs $\alpha x \bar{L}$ and $\beta(1-x) \bar{L}$ of sectors $A$ and $B$, respectively. Finally, the parameter $\theta>0$ represents the decay rate of $P$.

Following previous contributions in the literature (e.g. Ikefuji and Horii, 2012; Rezai et al., 2012; Hackett and Moxnes, 2015; Bretschger, 2017; Dao et al., 2017; Bretschger and Pattakou, 2019), we assume a negative impact of pollution on the output of the economy. In particular, we assume that a share of the aggregate outputs must be used as defensive expenditures to repair the damages due to pollution $P$, and that the net aggregate outputs are given by:

$$
\begin{aligned}
& \Omega_{A}(P) \cdot Y_{A} \\
& \Omega_{B}(P) \cdot Y_{B}
\end{aligned}
$$

where $\Omega_{A}(P)$ and $\Omega_{B}(P)$ are the so-called "damage coefficients":5

$$
\begin{aligned}
& \Omega_{A}(P)=\frac{1}{1+\gamma P} \\
& \Omega_{B}(P)=\frac{1}{1+\delta P}
\end{aligned}
$$

The parameters $\gamma>0$ and $\delta>0$ measure the vulnerability to pollution of sectors $A$ and $B$, respectively. Notice that the damage coefficients are a decreasing function of the pollution level:

\footnotetext{
${ }^{5}$ Such coefficients are commonly used in the literature on climate change (cf., for instance, Golub and Toman, 2016; Bretschger and Pattakou, 2019) to measure the share of output that is actually available once the environmental damages are taken into account.
} 
the higher is the pollution level, the lower the level of these coefficients, and therefore the lower also the output and income level of the corresponding sector net of the defensive expenditures. The latter (i.e. the quantities of the outputs that are not actually available for consumption and/or investment being used as defensive expenditures) are obviously given by the difference between the aggregate (gross) output of each sector and the net output described above, namely, $\left[1-\Omega_{A}(P)\right] \cdot Y_{A}$ and $\left[1-\Omega_{B}(P)\right] \cdot Y_{B}$, where:

$$
\begin{aligned}
& 1-\Omega_{A}(P)=\frac{\gamma P}{1+\gamma P} \\
& 1-\Omega_{B}(P)=\frac{\delta P}{1+\delta P}
\end{aligned}
$$

We assume that the dynamics of the share $x$ of land used in sector $A$ depends on the average (net) productivities of land employed in sectors $A$ and $B$ :

$$
\begin{aligned}
& \Pi_{A}(x, P)=\frac{\Omega_{A}(P) Y_{A}}{x \bar{L}}=\frac{\alpha}{1+\gamma P} \\
& \Pi_{B}(x, P)=\frac{\Omega_{B}(P) Y_{B}}{(1-x) \bar{L}}=\frac{\beta}{1+\delta P}
\end{aligned}
$$

and is given by the well-known replicator equation (see, among others, Hofbauer and Sigmund, 1988; Weibull, 1995):

$$
\dot{x}=x\left[\Pi_{A}(x, P)-\bar{\Pi}(x, P)\right]
$$

where $\dot{x}$ indicates the time derivative of $x$, while:

$$
\bar{\Pi}(x, P)=x \Pi_{A}(x, P)+(1-x) \Pi_{B}(x, P)
$$

measures average land productivity of the economy. According to (5), the share $x$ of land allocated in sector $A$ increases if the average productivity $\Pi_{A}(x, P)$ in sector $A$ is higher than the economy-wide average productivity $\bar{\Pi}(x, P) \cdot{ }^{6}$ Vice versa if the opposite holds. It is easy to check that equation (5) can be rewritten as:

$$
\dot{x}=x(1-x)\left[\Pi_{A}(x, P)-\Pi_{B}(x, P)\right]
$$

\footnotetext{
${ }^{6}$ Very similar qualitative dynamics can be obtained by substituting the replicator equation (7) with every payoff-monotonic dynamics (see Weibull, 1995).
} 
According to (7), the value of $x$ increases (decreases) when $\Pi_{A}>\Pi_{B}\left(\Pi_{A}<\Pi_{B}\right)$, while $x$ remains constant when $\Pi_{A}=\Pi_{B}$. This specification mirrors the fact that, as in the real world, profitability and productivity are the main drivers of farmers' land use choices.

Variations in the share $x$ affect the pollution level $P$, while variations in $P$ affect the relative performance of sectors, and therefore the time evolution of $x$, according to the system:

$$
\begin{gathered}
\dot{x}=x(1-x)\left(\frac{\alpha}{1+\gamma P}-\frac{\beta}{1+\delta P}\right) \\
\dot{P}=\varepsilon \alpha x \bar{L}+\eta \beta(1-x) \bar{L}-\theta P
\end{gathered}
$$

defined in the set:

$$
\Phi=\{(x, P): 0 \leq x \leq 1, P \geq 0\}
$$

\section{Stationary states and global dynamics}

The stationary states of the dynamic system (8) are given by the intersection points, belonging to the set $\Phi$ (see (9)), between the straight line (22), where $\dot{P}=0$ and one of the straight lines $x=0, x=1$, and (19), where $\dot{x}=0$ (see Appendix). For a graphical representation see Fig. 1. For simplicity, we shall limit our analysis to the cases in which $\alpha \delta-\beta \gamma \neq 0$ and $\varepsilon \alpha-\eta \beta \neq 0$ hold. In this context, the following proposition holds:

Proposition 1 In the context in which $\alpha \delta-\beta \gamma \neq 0$ and $\varepsilon \alpha-\eta \beta \neq 0$ hold, the dynamic system

(8) admits at most three stationary states:

a) the point $\left(0, P_{0}\right)=\left(0, \frac{\eta \beta}{\theta} \bar{L}\right)$, where the land endowment $\bar{L}$ is entirely allocated to sector $B$;

b) the point $\left(1, P_{1}\right)=\left(1, \frac{\varepsilon \alpha}{\theta} \bar{L}\right)$, where the land endowment $\bar{L}$ is entirely allocated to sector $A$;

c) the point $(\widetilde{x}, \widetilde{P})=\left(\frac{\theta}{(\beta \eta-\alpha \varepsilon) \bar{L}}\left(\frac{\alpha-\beta}{\alpha \delta-\beta \gamma}+\frac{\beta \eta \bar{L}}{\theta}\right), \frac{\beta-\alpha}{\alpha \delta-\beta \gamma}\right)$, where both sectors $A$ and $B$ coexist.

The stationary states $\left(0, P_{0}\right)$ and $\left(1, P_{1}\right)$ always exist, while the stationary state $(\widetilde{x}, \widetilde{P})$ exists if and only if the following two conditions hold:

1) $\widetilde{P}>0$, that is, either condition (20) or condition (21) is satisfied; 
2) $1>\widetilde{x}>0$, that is, either $P_{0}<\widetilde{P}$ and $P_{1}>\widetilde{P}$ (in the context of condition (23)) or $P_{0}>\widetilde{P}$ and $P_{1}<\widetilde{P}$ (in the context of condition (24)) hold.

The following proposition deals with the stability properties of the stationary states $\left(0, P_{0}\right)$, $\left(1, P_{1}\right)$, and $(\widetilde{x}, \widetilde{P})$.

Proposition 2 According to the dynamic system (8), the stationary state $\left(0, P_{0}\right)$ is locally attractive if:

$$
\frac{\alpha}{1+\gamma P_{0}}-\frac{\beta}{1+\delta P_{0}}<0
$$

while it is a saddle point (with stable manifold lying in the straight line $x=0$ ) if the opposite of condition (10) holds.

The stationary state $\left(1, P_{1}\right)$ is locally attractive if:

$$
-\left(\frac{\alpha}{1+\gamma P_{1}}-\frac{\beta}{1+\delta P_{1}}\right)<0
$$

while it is a saddle point (with stable manifold lying in the straight line $x=1$ ) if the opposite of (11) holds.

The stationary state $(\widetilde{x}, \widetilde{P})$ is locally attractive if:

$$
(\varepsilon \alpha-\eta \beta)(\alpha \delta-\beta \gamma)<0
$$

while it is a saddle point if the opposite of (12) holds.

Proof. The Jacobian matrix of the system (8), evaluated at the stationary state $(\widetilde{x}, \widetilde{P})$, can be written as follows:

$$
J(\widetilde{x}, \widetilde{P})=\left(\begin{array}{cc}
0 & \widetilde{x}(1-\widetilde{x})\left[\frac{\beta \delta}{(1+\delta \widetilde{P})^{2}}-\frac{\alpha \gamma}{(1+\gamma \widetilde{P})^{2}}\right] \\
(\varepsilon \alpha-\eta \beta) \bar{L} & -\theta
\end{array}\right)
$$

where:

$$
\operatorname{Tr} J(\widetilde{x}, \widetilde{P})=-\theta<0
$$




$$
\operatorname{Det} J(\widetilde{x}, \widetilde{P})=-\widetilde{x}(1-\widetilde{x})\left[\frac{\beta \delta}{(1+\delta \widetilde{P})^{2}}-\frac{\alpha \gamma}{(1+\gamma \widetilde{P})^{2}}\right](\varepsilon \alpha-\eta \beta) \bar{L}
$$

Taking into account that, at $(\widetilde{x}, \widetilde{P})$, it holds $\alpha /(1+\gamma \widetilde{P})-\beta /(1+\delta \widetilde{P})=0$, and therefore $(1+\delta \widetilde{P}) /(1+\gamma \widetilde{P})=$ $\beta / \alpha$, we can write

$$
\begin{gathered}
\frac{\beta \delta}{(1+\delta \widetilde{P})^{2}}-\frac{\alpha \gamma}{(1+\gamma \widetilde{P})^{2}}= \\
=\frac{\beta}{(1+\delta \widetilde{P})} \frac{\delta}{(1+\delta \widetilde{P})}-\frac{\alpha}{(1+\gamma \widetilde{P})} \frac{\gamma}{(1+\gamma \widetilde{P})}= \\
=\frac{\beta}{(1+\delta \widetilde{P})}\left[\frac{\delta}{(1+\delta \widetilde{P})}-\frac{\gamma}{(1+\gamma \widetilde{P})}\right]= \\
=\frac{\beta}{(1+\delta \widetilde{P})^{2}}\left[\delta-\frac{1+\delta \widetilde{P}}{1+\gamma \widetilde{P}} \gamma\right]=\frac{\beta}{(1+\delta \widetilde{P})^{2}}\left(\delta-\frac{\beta}{\alpha} \gamma\right)
\end{gathered}
$$

According to (14), we have:

$$
\operatorname{sign}[\operatorname{Det} J(\widetilde{x}, \widetilde{P})]=\operatorname{sign}[-(\varepsilon \alpha-\eta \beta)(\alpha \delta-\beta \gamma)]
$$

Therefore, $(\widetilde{x}, \widetilde{P})$ is locally attractive if $(\varepsilon \alpha-\eta \beta)(\alpha \delta-\beta \gamma)<0$, while it is a saddle point if the opposite inequality holds. The Jacobian matrix evaluated at $\left(0, P_{0}\right)$ is:

$$
J\left(0, P_{0}\right)=\left(\begin{array}{cc}
\frac{\alpha}{1+\gamma P_{0}}-\frac{\beta}{1+\delta P_{0}} & 0 \\
(\varepsilon \alpha-\eta \beta) \bar{L} & -\theta
\end{array}\right)
$$

with eigenvalues $-\theta<0$ (in direction of the $P$ - axis) and $\frac{\alpha}{1+\gamma P_{0}}-\frac{\beta}{1+\delta P_{0}}$.

Analogously, the Jacobian matrix evaluated at $\left(1, P_{1}\right)$ is:

$$
J\left(1, P_{1}\right)=\left(\begin{array}{cc}
-\left(\frac{\alpha}{1+\gamma P_{1}}-\frac{\beta}{1+\delta P_{1}}\right) & 0 \\
(\varepsilon \alpha-\eta \beta) \bar{L} & -\theta
\end{array}\right)
$$

with eigenvalues $-\theta<0$ (in direction of the $P$-axis) and $-\left(\frac{\alpha}{1+\gamma P_{1}}-\frac{\beta}{1+\delta P_{1}}\right)$.

Note that condition (10) holds if $P_{0}<\widetilde{P}$, in the context $\alpha \delta-\beta \gamma>0$, and if $P_{0}>\widetilde{P}$, in the 
context $\alpha \delta-\beta \gamma<0$ (see (19) and (21)). Analogously, condition (11) holds if $P_{1}>\widetilde{P}$, in the context $\alpha \delta-\beta \gamma>0$, and if $P_{1}<\widetilde{P}$, in the context $\alpha \delta-\beta \gamma<0$.

The following proposition deals with the global dynamics of system (8).

Proposition 3 Every set $\Gamma \subset \Phi$ :

$$
\Gamma=\{(x, P): 0 \leq x \leq 1, \bar{P} \geq P \geq 0\}
$$

with $\bar{P}>\max \left\{P_{0}, P_{1}\right\}$, is positively invariant under the dynamic system (8). Furthermore, every trajectory starting outside it enters it in finite time.

Proof. To prove this proposition, remember that $\dot{x}=0$ holds for $x=0$ and $x=1$, therefore the sides of $\Gamma$ with $x=0$ and $x=1$ are invariant. Furthermore, every point $(x, \bar{P})$ lies above the isocline $\dot{P}=0$ (and consequently $\dot{P}<0$ at $(x, \bar{P})$ ) while every point $(x, 0)$ lies below the isocline $\dot{P}=0$ (and consequently $\dot{P}>0$ at $(x, 0)$ ). This proves the positive invariance of the set $\Gamma$. Since in $\Phi-\Gamma$ no stationary state exists, then by the Poincaré-Bendixson Theorem, every trajectory starting in $\Phi-\Gamma$ enters $\Gamma$ in finite time.

The existence of limit cycles around the internal stationary state $(\widetilde{x}, \widetilde{P})$ is excluded when it is a saddle point (according to the Index theory, see e.g., Lefschetz, 1977), while it cannot be excluded when $(\widetilde{x}, \widetilde{P})$ is locally attractive, in that the divergence $\frac{\partial \dot{x}}{\partial x}+\frac{\partial \dot{P}}{\partial P}$ is not always negative in the set $\Gamma$ (see, e.g., Giné, 2014).

\section{Dynamic regimes}

We can now identify all possible dynamic regimes that may be observed and we can discuss the conditions under which each of them emerges. We exclude trivial cases in which the internal stationary state $(\widetilde{x}, \widetilde{P})$ does not exist and, then, either $\left(0, P_{0}\right)$ or $\left(1, P_{1}\right)$ is globally attractive in the interior of the set $\Phi$. More precisely, the stationary state $\left(1, P_{1}\right)$ (the stationary state $\left.\left(0, P_{0}\right)\right)$ with complete specialization in sector $A(B)$ is globally attractive when sector $A(B)$ is, at the same time, less (more) vulnerable to environmental degradation and more (less) productive than $B(A)$, namely with $\alpha>\beta(\beta>\alpha)$ and $\gamma<\delta(\gamma>\delta)$. 
Moving on to the more interesting cases characterized by the existence of the internal stationary state $(\widetilde{x}, \widetilde{P})$, the analysis of the isoclines $\dot{x}=0, \dot{P}=0$ of Appendix and stability analysis of Section 3 allows us to introduce the following taxonomy:

- Case (i): sector $A$ is less vulnerable and more polluting than sector $B$; this case occurs if conditions (20) and (23) hold. In other words, the main weakness of sector $A$ is a low productivity, but it can rely on other strenghts. More precisely, sector $A$ is subject to low environmental constraints and it exerts strong crowding out pressure on sector $B$ through its environmental impact.

- Case (ii): sector $A$ is less vulnerable and less polluting than sector $B$; this case occurs if conditions (20) and (24) hold. The only difference with case (i) is in that expansion of sector $A$ does not led to a net increase in pollution and therefore it does not create a crowding out effect on sector $B$.

- Case (iii): sector $A$ is more vulnerable and more polluting than sector $B$; this case occurs if conditions (21) and (23) hold. This is the opposite to case (ii).

- Case (iv): sector $A$ is more vulnerable and less polluting than sector $B$; this case occurs if conditions (21) and (24) hold. This is the opposite to case (i).

In Cases (i) and (iv), illustrated in Figs. 1(a) and 1(d), respectively, the stationary states $\left(0, P_{0}\right)$ and $\left(1, P_{1}\right)$ are locally attractive, while the internal stationary state $(\widetilde{x}, \widetilde{P})$ is a saddle point. The stable manifold of $(\widetilde{x}, \widetilde{P})$, represented in blue, separates the basins of attraction of $\left(0, P_{0}\right)$ and $\left(1, P_{1}\right)$. Therefore, bistable regimes are observed and the economy tends to specialise either in $A$ or in sector $B$, according to the starting point $(x(0), P(0))$. In Cases (ii) and (iii), the two sectors tend to coexist (Figs. 1(b) and $1(\mathrm{c}))^{7}$ More specifically, the stationary states $\left(0, P_{0}\right)$ and $\left(1, P_{1}\right)$ are saddle points, while the internal stationary state $(\widetilde{x}, \widetilde{P})$ is attractive. Fig. 1 illustrate numerical simulations of dynamics in cases (i)-(iv), respectively. In these figures, attractive stationary states are marked by full dots $(\bullet)$ and saddle points by squares ( $\square$ ).

\footnotetext{
${ }^{7}$ Notice that sector coexistence would be more likely to occur if variable prices and decreasing returns to scale were assumed since the marginal productivity of land in a given sector would decrease and its price would increase as the economy moves toward full specialization in that sector, thus pushing the system back toward the opposite sector. However, even under these assumptions, the dynamics of the model can still generate full specialization. Indeed, the only way to ensure sector coexistence is by assuming Inada conditions so that land productivity goes to infinity as land use goes to zero.
} 


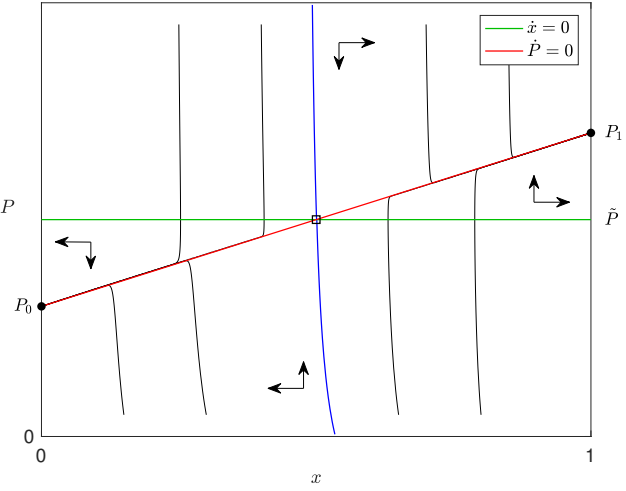

(a) Case (i).

Parameter values: $\alpha=1, \beta=2, \gamma=1, \delta=3, \varepsilon=3.5$, $\eta=0.75, \theta=2.5, \bar{L}=1$.

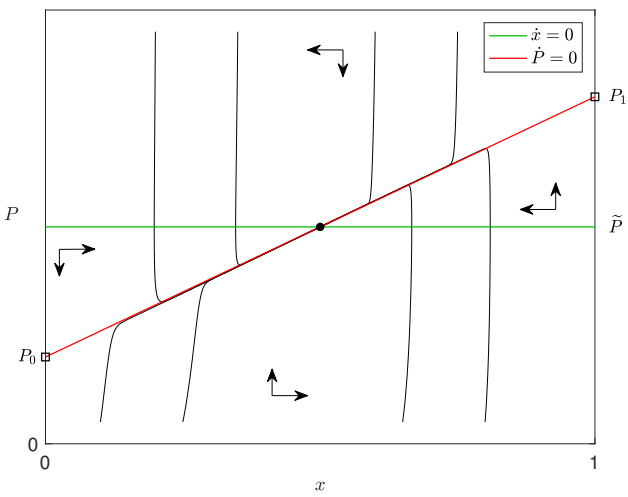

(c) Case (iii).

Parameter values: $\alpha=2, \beta=1, \gamma=3, \delta=1, \varepsilon=2$, $\eta=1, \theta=2.5, \bar{L}=1$.

Fig. 1. Dynamic regimes.

Legend: • sinks, $\square$ saddle points.

\subsection{Average land productivity of the economy in the scenarios (i)-(iv)}

In this section we compare the average land productivity of the economy (see (6)):

$$
\bar{\Pi}(x, P)=x \Pi_{A}(x, P)+(1-x) \Pi_{B}(x, P)
$$


evaluated at the stationary states $\left(0, P_{0}\right),\left(1, P_{1}\right)$, and $(\widetilde{x}, \widetilde{P})$ :

$$
\begin{array}{r}
\bar{\Pi}\left(0, P_{0}\right)=\Pi_{B}\left(0, P_{0}\right)=\frac{\beta}{1+\delta P_{0}} \\
\bar{\Pi}\left(1, P_{1}\right)=\Pi_{A}\left(1, P_{1}\right)=\frac{\alpha}{1+\gamma P_{1}} \\
\bar{\Pi}(\widetilde{x}, \widetilde{P})=\Pi_{A}(\widetilde{x}, \widetilde{P})=\Pi_{B}(\widetilde{x}, \widetilde{P})=\frac{\alpha}{1+\gamma \widetilde{P}}=\frac{\beta}{1+\delta \widetilde{P}}
\end{array}
$$

From which $\bar{\Pi}\left(0, P_{0}\right)<\bar{\Pi}(\widetilde{x}, \widetilde{P})<\bar{\Pi}\left(1, P_{1}\right)$ if and only if:

$$
\frac{\beta}{1+\delta P_{0}}<\frac{\alpha}{1+\gamma \widetilde{P}}=\frac{\beta}{1+\delta \widetilde{P}}<\frac{\alpha}{1+\gamma P_{1}}
$$

and $\bar{\Pi}\left(0, P_{0}\right)>\bar{\Pi}(\widetilde{x}, \widetilde{P})>\bar{\Pi}\left(1, P_{1}\right)$ if and only if:

$$
\frac{\beta}{1+\delta P_{0}}>\frac{\alpha}{1+\gamma \widetilde{P}}=\frac{\beta}{1+\delta \widetilde{P}}>\frac{\alpha}{1+\gamma P_{1}}
$$

Condition (15) holds if $P_{0}>P_{1}$; conversely, condition (16) holds if $P_{0}<P_{1}$. The following proposition summarizes these results.

Proposition 4 In Cases (i) and (iii), where condition (23) holds, the stationary states are ranked by the inequality:

$$
\bar{\Pi}\left(0, P_{0}\right)>\bar{\Pi}(\widetilde{x}, \widetilde{P})>\bar{\Pi}\left(1, P_{1}\right)
$$

In Cases (ii) and (iv), where condition (24) holds, the stationary states are ranked by the inequality:

$$
\bar{\Pi}\left(0, P_{0}\right)<\bar{\Pi}(\widetilde{x}, \widetilde{P})<\bar{\Pi}\left(1, P_{1}\right)
$$

The sectoral land allocation which ensures the highest level of average land productivity (and therefore also the highest total value output) is the one associated with lowest total pollution burden. In other words, the stationary states $\left(0, P_{0}\right),\left(1, P_{1}\right)$, and $(\widetilde{x}, \widetilde{P})$ can be ordered by average land productivity according to their inverse order by level of pollution $P$. The highest land productivity is reached when the economy is able to specialize in the clean sector ( $A$ in cases (ii) and (iv) or $B$ in cases (i) and (iii)). 


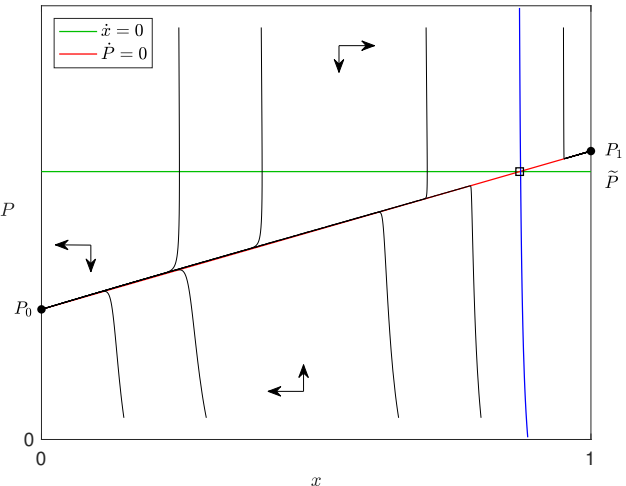

(a) $\alpha=0.95$.

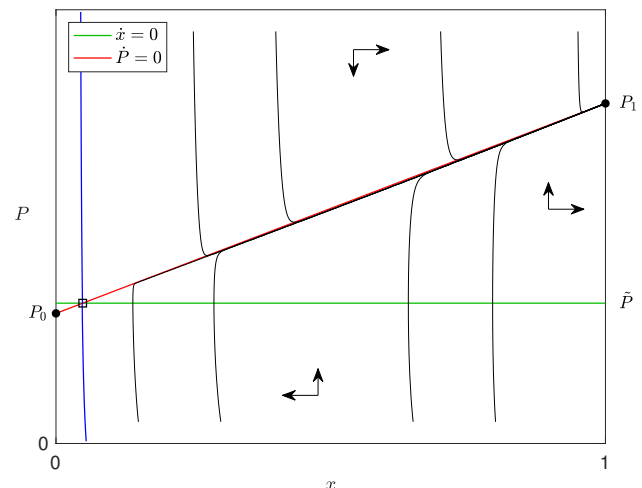

(b) $\alpha=1.12$.

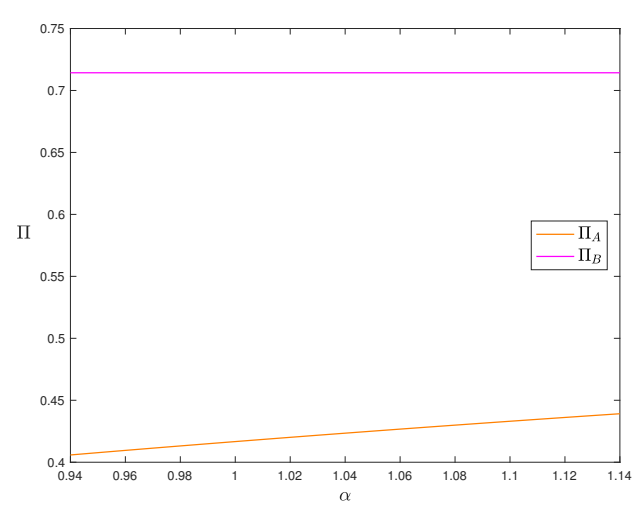

(c) Land average productivity.

Fig. 2. Case (i): basins of attraction for low (a) and high (b) value of $\alpha$, and land average productivity (c) of boundary stationary states.

Parameter values: $\beta=2, \gamma=1, \delta=3, \varepsilon=3.5, \eta=0.75, \theta=2.5, \bar{L}=1$.

Legend: • sinks, $\square$ saddle points.

\section{Simulations}

\subsection{Change in output elasticity with respect to land}

This section assesses the impact of an exogenous change in parameters representing output elasticity with respect to land ( $\alpha$ in sector $A$ and $\beta$ in sector $B$ ) on stationary states, their basins of attraction and average land productivity. The aim is to understand how the usually positive effects of increased productivity change once environmental dimensions are taken into account. Cases (i) and (iv) are symmetrical, as well all cases (ii) and (iii). For the sake of simplicity, we 


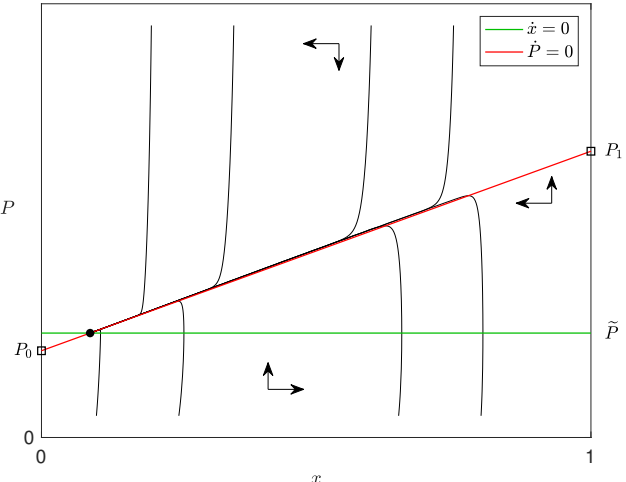

(a) $\alpha=1.65$.

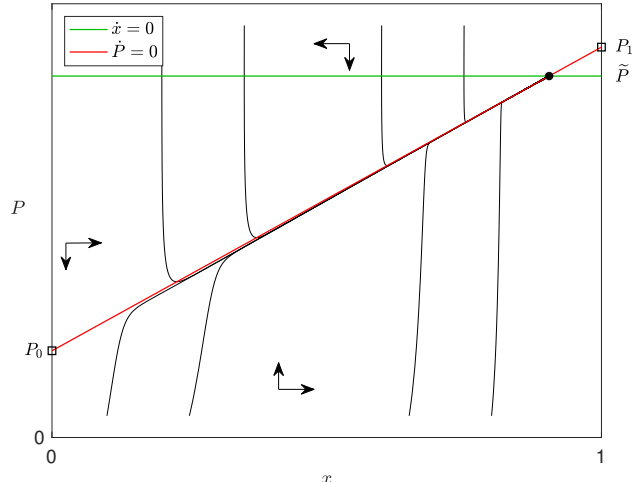

(b) $\alpha=2.25$.

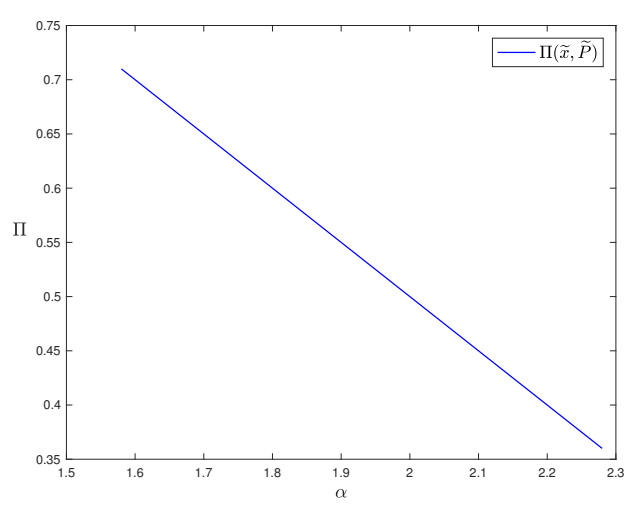

(c) Land average productivity.

Fig. 3. Case (iii): basins of attraction for low (a) and high (b) value of $\alpha$, and land average productivity (c) of inner stationary state.

Parameter values: $\beta=1, \gamma=3, \delta=1, \varepsilon=2, \eta=1, \theta=2.5, \bar{L}=1$.

Legend: • sinks, $\square$ saddle points.

narrow the analysis on cases (i) and (iii), in which sector $A$ can be labelled as "dirty" agriculture subsector. Cases (ii) and (iv) will produce exactly opposite results.

If the dirty sector $A$ experiences an exogenous growth in output elasticity with respect to land $\alpha$ under the bistable dynamic regime (i), the effect is an expansion of basin of attraction of the stationary state $\left(1, P_{1}\right)$ where land endowment is entirely allocated to the dirty sector $A$ (see Figs. 2(a) and 2(b)). In other words, the economy is more likely to follow a welfarereducing specialization characterized by lower farmers' average revenues (see Fig. 2(c)) than in the alternative attractive stationary state $\left(0, P_{0}\right)$. 


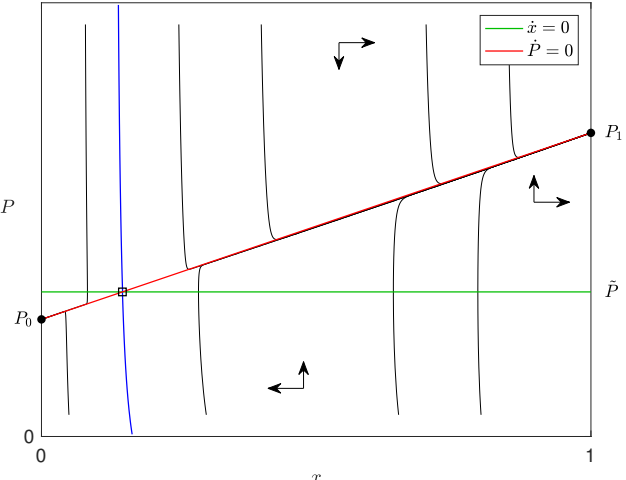

(a) $\beta=1.8$.

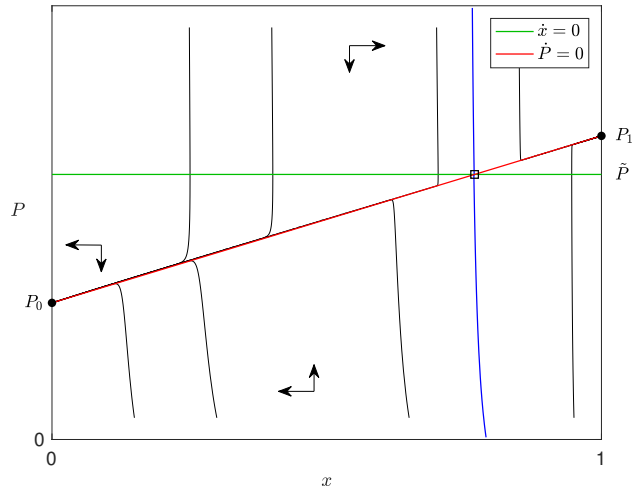

(b) $\beta=2.1$.

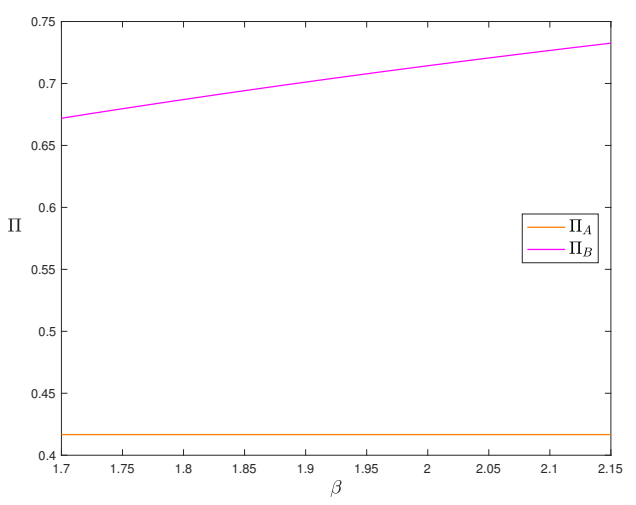

(c) Land average productivity.

Fig. 4. Case (i): basins of attraction for low (a) and high (b) value of $\beta$, and land average productivity (c) of boundary stationary states.

Parameter values: $\alpha=1, \gamma=1, \delta=3, \varepsilon=3.5, \eta=0.75, \theta=2.5, \bar{L}=1$.

Legend: • sinks, $\square$ saddle points.

Fig. 3 shows the effect of an exogenous growth in output elasticity with respect to land $\alpha$ in case (iii), namely when sector $A$ is, not only more polluting, but also more productive and more environmentally vulnerable than sector $B$. In this scenario, the effect is a shift of the internal globally attractive stationary state $(\widetilde{x}, \widetilde{P})$ towards a larger land share allocated to the dirty sector A. Despite the exogenous productivity gain, also in this case the result is a decline in overall farmers' revenue as illustrated in Fig. 3(c) reporting a reduction in average land productivity.

Opposite results are produced by an exogenous increase in $\beta$ which represents the output elasticity with respect to land in sector $B$, namely the "clean" sector under the dynamic regimes 


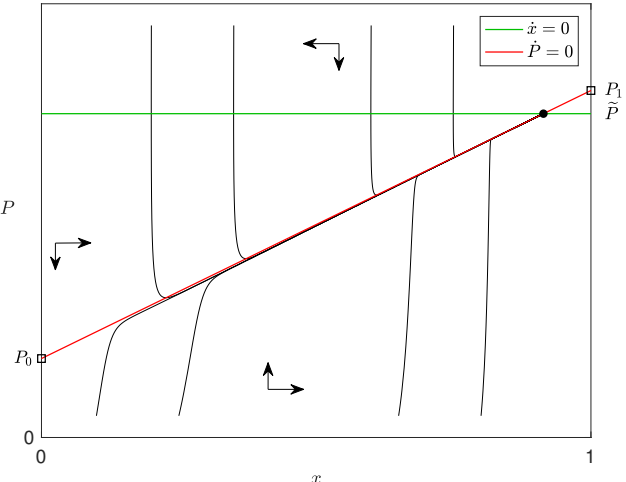

(a) $\beta=0.91$.

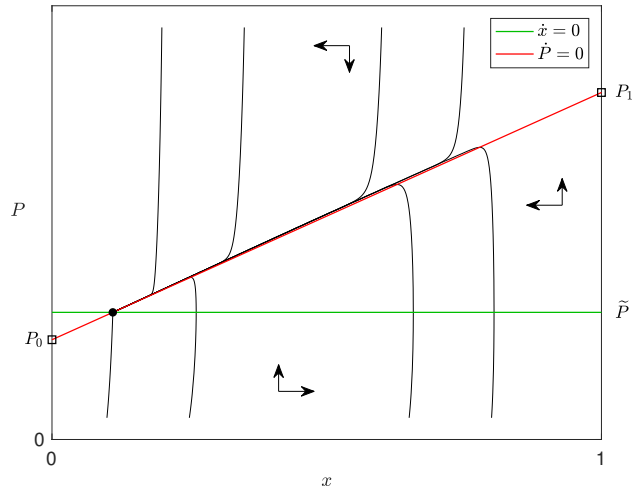

(b) $\beta=1.15$.

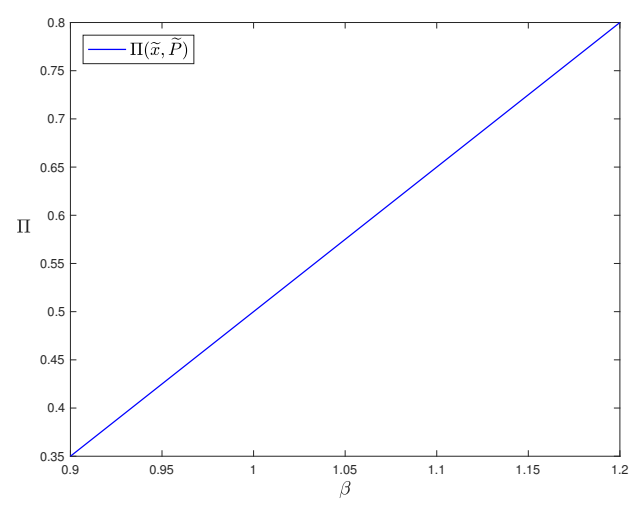

(c) Land average productivity.

Fig. 5. Case (iii): basins of attraction for low (a) and high (b) value of $\beta$, and land average productivity (c) of inner stationary state.

Parameter values: $\alpha=2, \gamma=3, \delta=1, \varepsilon=2, \eta=1, \theta=2.5, \bar{L}=1$.

Legend: • sinks, $\square$ saddle points.

(i) and (iii) (see Figs. 4 and 5).

\subsection{Change in degree of vulnerability to environmental degradation}

We can now give a closer look at the role of vulnerability to pollution, a key driver of agriculture development in territory exposed to environmental externalities. We focus again on dynamic regimes (i) and (iii) in which the sector $A$ is characterized by a higher pollution intensity than sector $B$. If the economy is under monostable dynamic regime (case (iii), see Figs. 6(c) and 6(d)), 


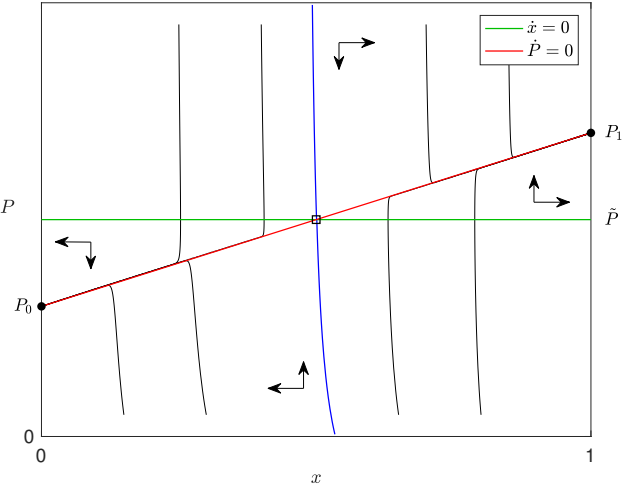

(a) Case (i), starting framework.

Parameter values: $\alpha=1, \beta=2, \gamma=1, \delta=3, \varepsilon=3.5$, $\eta=0.75, \theta=2.5, \bar{L}=1$.

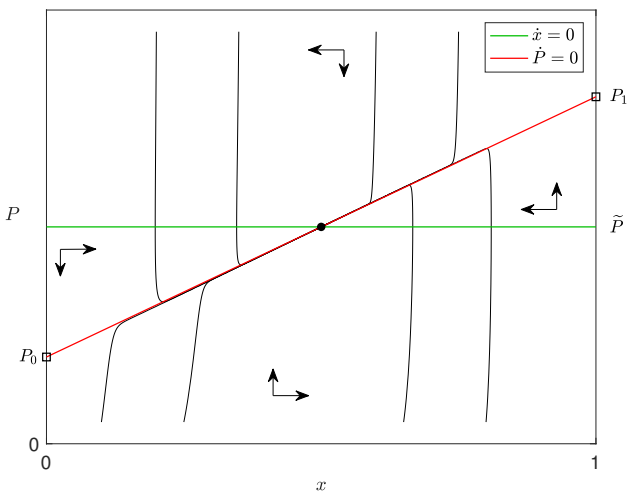

(c) Case (iii), starting framework.

Parameter values: $\alpha=2, \beta=1, \gamma=3, \delta=1, \varepsilon=2$, $\eta=1, \theta=2.5, \bar{L}=1$.

Fig. 6. Vulnerabilities.

Legend: • sinks, $\square$ saddle points.

as long as the clean sector becomes less resilient to pollution (i.e. $\delta$ increases), the globally attractive equilibrium $(\widetilde{x}, \widetilde{P})$ shifts and the corresponding transition is associated with an increase in the pollution stock and in the share of land used in the dirty sector. If the economy belongs to a bistable dynamic regime (i), as shown in Figs. 6(a) and 6(b), an exogenous growth of vulnerability of the clean sector leads to a shrinking basin of attraction of the Pareto-superior stationary state. In both cases, the final outcome is a welfare loss. 


\section{Interpretation of the results}

The results of the analysis provide interesting insights. In order to give more substance to the results, we organise the discussion in a question-answer format.

1) Output value is positively associated with output elasticity with respect to land and negatively associated with environmental vulnerability. Specialization in farming activities with high elasticity with respect to land (i.e. high land productivity) and low sensitivity to environmental pressure would be the ideal land use. Unfortunately, farming subsectors rarely present these two features at the same time. In case of trade-off between land productivity and resilience (as in all considered dynamic regimes (i)-(iv)), should pattern of land allocation move towards the most productive sector or towards the most resilient one? The answer is "it depends". Our results show that any structural change characterized by an increase in land share allocated to the cleanest subsector leads to an increase in average land productivity and this is true in all scenarios (i)-(iv). Let consider again cases (i) and (iii): in both regimes, $A$ is more polluting than $B$. In case (i), sector $A$ has the advantage of a low environmental vulnerability and the disadvantage of a low productivity. The dynamic regime is bistable and the stationary state $\left(0, P_{0}\right)$ with a complete specialization in the cleaner sector $B$ ensures a higher total output value than the stationary state $\left(1, P_{1}\right)$ with a complete specialization in sector $A$. In case (iii), sector $A$ has the advantage of a high productivity and the disadvantage of a high vulnerability to pollution. In this regime, the internal stationary state $(\widetilde{x}, \widetilde{P})$ is globally attractive. Also in this case, the stationary state $\left(0, P_{0}\right)$ outperforms the other stationary states in terms of output value, but it is unstable. Structural changes can occur when variations in parameter values make internal stationary state $(\widetilde{x}, \widetilde{P})$ move. Simulations on parameter $\alpha$ are an example: an increase in $\alpha$ generates a structural change towards a larger land share of sector $A$ and the result is a lower average land productivity indicating the farmers' welfare would instead improve along a shift towards the clean sector $B$. These results clearly indicate that the first criterion in land allocation choices should be the minimization of environmental impact, namely moving land allocation towards the sector with lower pollution intensity.

2) In many cases, territorial development tends to landscape uniformity and specialization. In 
principle, an alternative and successful land use pattern would be a balanced coexistence between more productive and polluting agricultural activities with less productive but cleaner ones. Why does this scenario rarely occur? In our model, this dynamic regime is admissible only in cases (ii) and (iii), that is when environmental impact intensity is positively related to pollution vulnerability. In the real world, this condition rarely holds and, as result, a non transient and stable coexistence of farming sectors with different environmental burden and constraints is quite infrequent. Take the example of organic and conventional farming: in absence of a stable policy support, organic farming struggles to gain market shares. Organic agriculture is overall more "integrated" in surrounding ecosystems from which it derives several natural resource inputs and environmental services. It is therefore, not only less polluting but also more sensitive to pollution than conventional farming which relies more on chemical and man-made "substitutes". The interactions between organic and convential farming are therefore better described by the dynamic regime (i) or (iv) than (ii) and (iii). The same observation applies to GMO and GMO-free crops. GM crops generate heavy externalities on GMO-free cultivations, in particular on organic production, which are exposed to the risk of "contamination". Also convential farmers can be damaged in case of contamination, especially if transition to GM seeds is accompanied by a change in farming practices. The so called "soybeanization" of Argentinian agriculture, for instance, started in the mid-1990s with the introduction of the transgenic Roundup Ready (RR) soybean together with a technological package of chemical fertilization and intensive use of the herbicide glyphosate (the new GM soy variety is resistant to it) with heavy detrimental impacts on human health of local population, soil quality, biodiversity and forest coverage (Leguizamón, 2014; Phélinas and Choumert, 2017). The introduction of GM soybean seems to portray the effect of a sudden increase in land productivity under dynamic regime (i) (rather than under regime (iii)) which leads the economy towards the specialization in the sector with higher environmental impact (see paragraph on simulations) and towards an overall welfare loss. Cultivation of GM soybeans, in fact, has expanded at the expense of cattle, maize, sunflower, and wheat production and its growth has been so rampant that now it accounts for $60 \%$ of total land cultivated in the country (Phélinas and Choumert, 2017). However, despite their current economic superiority in terms of profits and produc- 
tivity compared to non-GMO, environmental implications of GM soybean make economic and environmental sustainability of this process of specialization less certain, if not illusory.

3) What is the role for policy? We can give only an indirect answer as policy instruments are not included in the model. However, the results of the analysis and of simulations produce interesting insights. Agents tend to choose land use allocation merely looking at productivity without fully considering environmental burden and constraints generated by the choices taken by all agents. Thus, uncoordinated agents' choices are unlikely to converge to the land use composition corresponding to the Pareto superior stationary state. This equilibrium is unstable (cases (ii) and (iii)) or can be reached only if the economy starts from a situation in which the cleaner sector already accounts for a sufficiently high land share (case (i) and (iv)). The first normative implication is that, in order to avoid welfare-reducing and Pareto-dominated land allocation configurations, policy should focus on incentives to invest in low impact farming activities, even when they are characterized by a low land productivity. For this purpose, several policy instruments can be used depending on the specific case and corresponding dynamic regime taken into account. For instance, if the two sectors coexist at the equilibrium (i.e. there is a globally attractive inner stationary state), a differentiated tax rate on the production of the two sectors may modify the relative payoffs of the two sectors, reducing the relative profits of the more polluting sector and thus shifting economic activity towards the cleaner sector. If, on the contrary, a bistable dynamic regime emerges from the analysis leading to full specialization in one sector, then policy-makers might consider intervening on the initial land allocation between the two sectors (e.g. establishing the maximum share of total available land that can be allocated to the clean/dirty sector) so that the economic system lies in the attraction basin of the socially optimal stationary state. Other possible policy instruments that may drive the economic pathway towards a Pareto-superior equilibrium are public investments in the cleaner sector that reduce its vulnerability to environmental degradation and/or increase its land productivity.

Secondly, policy makers should prevent sudden exogenous changes which risk changing land use pathway of the economy bringing it along an inefficient and self-reinforcing specializa- 
tion in polluting agricultural subsector. Interestingly this may occur also as consequence of positive economic shocks, as in the case of an increase in land productivity of the dirty sector (see the example of the "soybeanization" of Argentina).

A negative reversal of land use pattern can be caused also by other type of shocks such as an increase in vulnerability of agricultural activities with lower environmental impact. In this case, an illustrative real-world case is represented, to some extent, by the rapid growth of commercial shrimp culture, experienced by southwestern coastal areas of Bangladesh over the last 30 years. Shrimp farming expansion has been driven not only by growing demand and policy support, but also by its greater suitability to changing environmental ecosystems. The construction of several mega-projects which have transformed water resource systems, together with sea level rise and storm surges, have dramatically modified environmental conditions (Pouliotte et al., 2009). Land-based crops have become more vulnerable to salt water intrusions. Production system in several villages have undertaken a transition from a specialization in rice and other traditional crops to salt water shrimp farming which, in turns, has several environmental implications, from destruction of mangroves, increased salinization and intensive use of chemicals (Islam and Yasmin, 2017). Using our model wording, crop production and shrimp colture can be represented, respectively, as sector $B$ and sector $A$. The economy is characterized by the bistable dynamic regime (i) as sector $B$ is less polluting and more vulnerable than sector $A$. This "blue revolution", as often referred to, in our model can be interpreted as the result of a sudden increase in parameter $\delta^{8}$ so that the economy, starting in the basin of attraction of stationary state $\left(0, P_{0}\right)$, ends up into the basin of attraction of $\left(1, P_{1}\right)$ with complete specialization in the dirty sector $A$. In other words, this exogenous shock made the economy "jump" on trajectories approaching a different stationary state and radically changed land use pattern following a process of welfare reduction, self-reinforcing specialization in polluting activities and crowding out of traditional crop farming. Indeed, despite the immediate economic benefits of specialization in shrimp farming, increasing concerns have been expressed on environmental and socioeconomic sustainability (Deb, 1998; Pouliotte et al., 2009; Paul and Vogl, 2011) of shrimping

\footnotetext{
${ }^{8}$ This interpretation obviously does not need to exclude the role of other determinants such as policy actions, growing international demand for shrimps, big investors' interest for profit opportunities in export markets.
} 
culture itself and of overall territorial development of Bangladesh's coastal areas.

\section{Conclusions}

Agricultural development usually means choosing the best performing agriculture activity in terms of land productivity. We have revised this apparent tautology by introducing two main elements in a evolutionary model of sectoral land allocation:

1) environmental constraints that, in turn, depend on pollution intensity and vulnerability.

2) interactions between different farming activities. Each individual's decision on the preferred agricultural activity does not occur in an impermeable setting. In contrast, it is influenced by land use choices of all farmers.

We show that multiple dynamic regimes are possible. However, conditions for the existence of bistable dynamic regimes seem to be more consistent with recurrent features of real-world interactions between agricultural subsectors. We find that in this context, the economy is at risk of following a land use pattern associated with increasing environmental degradation and welfare decline even if this development path occurs along a specialization in the sector with higher land productivity or even if the transition is prompted by a positive shock such as an increase in land productivity.

If policy goal is to approach the economy to the stationary states at which well-being is greater, policy action needs to promote the cleaner sector in order to head land use sectoral composition towards low levels of pollution. Possible instruments for this purpose include differentiated (sector-based) taxes/subsidies, land use planning establishing minimum/maximum land quotas that can be devoted to different sectors and public investments in adaptation activities reducing the vulnerability of clean sectors to environmental degradation. These and other sector-specific measures can be very effective in driving structural change, as shown by the recent increase in organic farming in the European Union where it is estimated that 56 per cent of producers benefited from various support measures by the European Common Agricultural Policy (European Commission, 2013, 2019).

The present model deliberately proposes a very simple analytical framework to identify a few 
possible causal links that may generate undesirable outcomes. Future research, however, should examine whether and how results may differ by relaxing some of the underlying simplifying assumptions. For instance, it would be interesting to see how results may change assuming different and variable willingness to pay for the goods produced by each sector. Indeed, a few people may initially have higher willingness to pay for organic farming than for conventional farming with pesticides. However, individual willingness to pay and the share of environmentalfriendly consumers may be endogenously affected by the other consumers' preferences and/or by policy interventions (e.g. a better information campaign on health consequences of different products). This endogenous mechanism would not probably alter the main dynamic regimes observed in the model, but it could generate price changes over time, affecting the value of the stationary state and their basins of attraction. Finally, while in the model we focus on a unique kind of pollutant, it would be interesting to investigate what would happen if we assume that different sectors may generate different pollutants and/or different kinds of environmental degradation. We leave these analyses to future extensions of the model.

\section{Appendix}

Isoclines $\dot{x}=0$ and $\dot{P}=0$

According to (8), $\dot{x}=0$ holds for $x=0,1$ and for:

$$
P=\widetilde{P}=\frac{\beta-\alpha}{\alpha \delta-\beta \gamma}
$$

if $\alpha \delta-\beta \gamma \neq 0^{9}$, where $\widetilde{P}>0$ if either condition:

$$
\alpha-\beta<0 \text { and } \alpha \delta-\beta \gamma>0
$$

or condition:

$$
\alpha-\beta>0 \text { and } \alpha \delta-\beta \gamma<0
$$

\footnotetext{
${ }^{9}$ When $\alpha \delta-\beta \gamma=0$, then it holds $\dot{x}>0$ (respectively, $\dot{x}=0$ and $\dot{x}<0$ ) if $\alpha-\beta>0$ (respectively, $\alpha-\beta=0$ and $\alpha-\beta<0$ ), whatever the value of $P$ is.
} 
holds. Notice that condition (20) may be satisfied only if $\gamma<\delta$, namely if sector $A$ is less vulnerable to pollution than sector $B$. This means that in a context of heavy pollution, the sector (in this case $A$ ) which is less sensitive to environmental pressure is able to reach stronger profitability performance compared the other one $(B)$ even it exhibits lower land productivity. When condition (20) applies, then above (below) the straight line (19), in the plane $(x, P)$, it holds $\dot{x}>0(\dot{x}<0)$. This means that the share of land allocated to sector $A$ tends to increase for high levels of pollution (for a graphical representation see Fig. 1 reporting different dynamic regimes which will be discussed later in Section 4).

Conversely, condition (21) may be satisfied only if $\gamma>\delta$, namely if sector $A$ is more vulnerable to pollution than sector $B$. When condition (21) applies, then above (below) the straight line (19), in the plane $(x, P)$, it holds $\dot{x}<0(\dot{x}>0)$. In this context, the share of land allocated to sector $A$ decreases for high levels of pollution $P$.

Analogously, according to (8), $\dot{P}=0$ holds for:

$$
P=\frac{\eta \beta \bar{L}}{\theta}+(\varepsilon \alpha-\eta \beta) \frac{\bar{L}}{\theta} x
$$

It holds $\dot{P}<0$ above the straight line (22), while $\dot{P}>0$ below it. The straight line (22) has positive slope (i.e., along it, $P$ increases if $x$ increases, see Fig. 1(a) and Fig. 1(c)) if:

$$
\varepsilon \alpha-\eta \beta>0
$$

while it has negative slope (see Fig. 1(b) and Fig. 1(d)) if:

$$
\varepsilon \alpha-\eta \beta<0
$$

We can observe that $\varepsilon \alpha$ and $\eta \beta$ represent the environmental impact of one land unit employed in sector $A$ and sector $B$, respectively. Thus if condition (23) holds, then shifting the use of one land unit from sector $A$ to sector $B$ results in a net decrease in the pollution stock.

Moreover, for $x=0$, equation (22) gives:

$$
P=P_{0}=\frac{\eta \beta}{\theta} \bar{L}>0
$$


while, for $x=1$, it gives:

$$
P=P_{1}=\frac{\varepsilon \alpha}{\theta} \bar{L}>0
$$

\section{References}

Adelman, I., 1984. Beyond export-led growth. World Development 12 (9), 937-949.

Arndt, C., Benfica, R., Tarp, F., Thurlow, J., Uaiene, R., 2010. Biofuels, poverty, and growth: a computable general equilibrium analysis of Mozambique. Environment and Development Economics 15 (1), 81-105.

Bajželj, B., Richards, K. S., 2014. The positive feedback loop between the impacts of climate change and agricultural expansion and relocation. Land 3 (3), 898-916.

Baudron, F., Giller, K. E., 2014. Agriculture and nature: trouble and strife? Biological Conservation $170,232-245$.

Bellemare, M. F., 2012. As you sow, so shall you reap: the welfare impacts of contract farming. World Development 40 (7), 1418-1434.

Bengtsson, J., Ahnström, J., Weibull, A.-C., 2005. The effects of organic agriculture on biodiversity and abundance: a meta-analysis. Journal of Applied Ecology 42 (2), 261-269.

Bretschger, L., 2017. Climate policy and economic growth. Resource and Energy Economics 49, 1-15.

Bretschger, L., Pattakou, A., 2019. As bad as it gets: how climate damage functions affect growth and the social cost of carbon. Environmental and Resource Economics 72 (1), 5-26.

Bretschger, L., Suphaphiphat, N., 2014. Effective climate policies in a dynamic North-South model. European Economic Review 69, 59-77.

Bretschger, L., Valente, S., 2011. Climate change and uneven development. Scandinavian Journal of Economics 113 (4), 825-845.

Brittain, C., Williams, N., Kremen, C., Klein, A.-M., 2013. Synergistic effects of non-apis bees and honey bees for pollination services. Proceedings of the Royal Society of London B 280 (1754), 20122767.

Christiaensen, L., Demery, L., Kuhl, J., 2011. The (evolving) role of agriculture in poverty reduction - an empirical perspective. Journal of Development Economics 96 (2), 239-254.

Dao, N. T., Burghaus, K., Edenhofer, O., 2017. Self-enforcing intergenerational social contracts for Pareto improving pollution mitigation. Environmental and Resource Economics 68 (1), 129-173.

De Janvry, A., Sadoulet, E., 2010. Agricultural growth and poverty reduction: additional evidence. World Bank Research Observer 25 (1), 1-20.

De Janvry, A., Sadoulet, E., 2017. Develpment economics: theory and practice. Routledge, London and New York.

Deb, A. K., 1998. Fake blue revolution: environmental and socio-economic impacts of shrimp culture in the coastal areas of Bangladesh. Ocean \& Coastal Management 41 (1), 63-88. 
Diao, X., Thurlow, J., Benin, S., Fan, S., 2012. Strategies and priorities for African agriculture: economywide perspectives from country studies. International Food Policy Research Institute.

European Commission, 2013. Overview of CAP Reform 2014-2020. Agricultural Policy Perspectives Brief.

European Commission, 2019. Organic farming in the EU. EU Agricultural Markets Briefs.

Gabriel, D., Tscharntke, T., 2007. Insect pollinated plants benefit from organic farming. Agriculture, Ecosystems \& Environment 118 (1-4), 43-48.

Gigante Carvalheiro, L., Veldtman, R., Shenkute, A. G., Tesfay, G. B., Pirk, C. W. W., Donaldson, J. S., Nicolson, S. W., 2011. Natural and within-farmland biodiversity enhances crop productivity. Ecology Letters 14 (3), 251-259.

Giné, J., 2014. Non-existence of limit cycles for planar vector fields. Electronic Journal of Differential Equations 2014 (75), 1-8.

Golub, A., Toman, M., 2016. Climate change, industrial transformation, and "environmental growth traps". Environmental and Resource Economics 63 (2), 249-263.

Hackett, S. B., Moxnes, E., 2015. Natural capital in integrated assessment models of climate change. Ecological Economics 116, 354-361.

Hofbauer, J., Sigmund, K., 1988. The theory of evolution and dynamical systems. Cambridge University Press.

Ikefuji, M., Horii, R., 2012. Natural disasters in a two-sector model of endogenous growth. Journal of Public Economics 96, 784-796.

IPCC Glossary, 2014. Climate Change 2014 Report Fifth Assessment Report. Intergovernmental Panel On Climate Change.

Islam, M., Yasmin, R., 2017. Impact of aquaculture and contemporary environmental issues in Bangladesh. International Journal of Fisheries and Aquatic Studies 5 (4).

Johnston, B. F., Mellor, J. W., 1961. The role of agriculture in economic development. American Economic Review 51 (4), 566-593.

Lefschetz, S., 1977. Differential equations: geometric theory. Dover Publication, New York, US.

Leguizamón, A., 2014. Modifying Argentina: GM soy and socio-environmental change. Geoforum $53,149-160$.

Lichtenberg, E., 2002. Agriculture and the environment. Handbook of Agricultural Economics $2,1249-1313$.

Ligon, E., Sadoulet, E., 2018. Estimating the relative benefits of agricultural growth on the distribution of expenditures. World Development 109, 417-428.

Paul, B. G., Vogl, C. R., 2011. Impacts of shrimp farming in Bangladesh: challenges and alternatives. Ocean \& Coastal Management 54 (3), 201-211.

Pelletier, N., Tyedmers, P., 2010. Forecasting potential global environmental costs of livestock production 2000-2050. Proceedings of the National Academy of Sciences 107 (43), 1837118374. 
Phélinas, P., Choumert, J., 2017. Is GM soybean cultivation in Argentina sustainable? World Development 99, 452-462.

Pouliotte, J., Smit, B., Westerhoff, L., 2009. Adaptation and development: livelihoods and climate change in Subarnabad, Bangladesh. Climate and Development 1 (1), 31-46.

Rauscher, M., Barbier, E. B., 2010. Biodiversity and geography. Resource and Energy Economics 32 (2), 241-260.

Rezai, A., Foley, D. K., Taylor, L., 2012. Global warming and economic externalities. Economic Theory 49 (2), 329-351.

Vardas, G., Xepapadeas, A., 2010. Model uncertainty, ambiguity and the precautionary principle: implications for biodiversity management. Environmental and Resource Economics 45 (3), 379 404.

Vogel, S. J., 1994. Structural changes in agriculture: production linkages and agricultural demand-led industrialization. Oxford Economic Papers 46 (1), 136-156.

Weibull, J., 1995. Evolutionary game theory. MIT Press. 